Preprint version, published (first online) in Synthese, Special Issue: Radical Views on Cognition.

Please refer to the published version: https://link.springer.com/article/10.1007/s11229-019-02253-2

\title{
An Ecological Approach to Disjunctivism
}

\section{Eros Moreira de Carvalho (UFRGS/CAPES/CNPq)}

Abstract: In this paper I claim that perceptual discriminatory skills rely on a suitable type of environment as an enabling condition for their exercise. This is because of the constitutive connection between environment and perceptual discriminatory skills, inasmuch as such connection is construed from an ecological approach. The exercise of a discriminatory skill yields knowledge of affordances of objects, properties, or events in the surrounding environment. This is practical knowledge in the first-person perspective. An organism learns to perceive an object by becoming sensitized to its affordances. I call this position ecological disjunctivism. A corollary of this position is that a case of perception and its corresponding case of hallucination-which is similar to the former only in some respects-are different in nature. I show then how the distinguishability problem is addressed by ecological disjunctivism.

Keywords: Discriminatory skills; ecological approach to perception; disjunctivism; affordances; practical knowledge.

\section{Introduction}

Gibson ended his paper “New Reasons for Realism”, published in Synthese, with a plea for help (1967, p. 172). He asked us to join him in the task of figuring out the epistemological consequences of his then new ecological view of perception. Responding to this call, I claim that disjunctivism and the ecological view of perception fit each other. ${ }^{1}$ The ecological view of perception is good for

1 In the same vein, La Favela and Chemero (2016) put forward an ecological account of visual illusions in support of a direct theory of perception. However, they do not seem to agree that disjunctivism and ecological psychology can form a good match. They suggest that disjunctivists "often engage in debates that discuss perception in traditional 
disjunctivism in providing a response to Tyler Burge's objection that disjunctivism is incompatible with science (2011, p. 43). ${ }^{2}$ Disjunctivism is not only compatible with but also supported by ecological psychology. The ecological view also helps disjunctivism to explain why an episode of perception and its corresponding episode of hallucination are different in nature. Due to the way an organism becomes sensitized to its environment, the perceptual skills of the organism can only be exercised in their proper environment. Whatever the response of the organism in an improper environment, it's not the outcome of the exercise of a perceptual skill or ability. As I will show, this ecological construal of perceptual skills helps disjunctivism to deal with the distinguishability problem. The problem here is that even if an episode of perception and its corresponding episode of hallucination are different in nature, as claimed by disjunctivists, this difference is not enough to explain how one can know that they are perceiving, rather than hallucinating-particularly if these episodes are indistinguishable from the first-person perspective. In the other direction, disjunctivism is good for ecological psychology in providing an epistemological framework within which Gibson’s claim that “perceiving is the simplest and best kind of knowing” (Gibson 2015, p. 251) can

terms” (2016, p. 71) and that ecological psychologist does not need to appeal to "disjunctive contents to explain” hallucinations (2016, p. 77). But the ecological psychologist appeals to disjunctive principles to explain hallucinations (2016, p. 71-72) and this precise point can be embedded in a disjunctive account of experience. Besides, as I will argue in Section 4, the ecological view of perception can benefit from a partnership with disjunctivism. Another interesting work that follows the same trajectory as this study is Beaton's (2016) proposal to read the sensorimotor theory of perception as a form of direct realism. There are relevant similarities between his results and those I advance in this paper, although we draw on different resources and take different paths. The present work may be seen as complementary in some respects to Beaton's work. He also claims that his position is both disjunctivist and direct realist (2016, p. 265). However, while he stresses the direct realist aspect of his position, I stress the disjunctivist aspect. We both sustain not only that an episode of perception and its corresponding episode of hallucination are different in nature but also that, given the proper circumstances, one can know whether one is perceiving or not. However, we have different approaches to introspection. He, relying on Shoemaker's work on introspection, maintains that one can have propositional knowledge of whether one is perceiving, while I, relying on ecological resources, maintain that one can have second-order practical knowledge of one's perceptual episodes. Another component of our works that is complementary is the attention I give to how the ecological approach to perception helps to clarify the notion of skill that underpins disjunctivism-at least the version I advocate-while Beaton gives more attention to how direct realism helps to clarify the philosophical consequences of the sensorimotor theory of perception.

2 According to Burge, the science of perception explains episodes of perception and episodes of illusion or hallucination based on a common factor. The same "quasi-deterministic laws between registration of proximal stimulation an the perceptual states" (2011, p. 44) explain how someone comes to have a perceptual or a hallucinatory state. The differences between theses states are due to distal inputs, "the causal chains that lead from the environment to the same registration of proximal stimulation.” (2011, p. 44) As, according to science, the same mechanism explains the occurrence of an episode of perception and an episode of hallucination, disjunctivism must be wrong since it rejects this fundamental and explanatory common factor. However, as I will show later, episodes of perception and of hallucination are not to be explained by the same principles or laws according to ecological psychology. Thus, Burge is wrong in rejecting disjunctivism only because of his restricted diet of sciences. 
be explained. By construing perceptual abilities as skills of access (Noë 2015, p. 1) or as capacities that provides us openness to the world (McDowell 1996, p. 111), we can explain why the process of picking up information in the environment provides us cognitive contact with (Millar 2007, p. 176) and therefore knowledge of the world.

In the next section I will outline Gibson's ecological approach to perception. Then, in Section 3, I argue that a discriminatory skill, as understood from an ecological approach, can only be exercised in its proper environment. In Section 4, I argue that this conception of discriminatory skills fits with a disjunctivist view of perception. Finally, in the last Section, I show how the proposed view deals with the distinguishability problem.

\section{The ecological approach to perception}

It has always been assumed that the senses were channels of sensation. To consider them as a system of perception, as this book proposes to do, may sound strange. But the fact is that there are two different meanings of the verb to sense, first, to detect something, and second, to have a sensation. When the senses are considered as perceptual systems the first meaning of the term is being used. (Gibson 1968, p. 1)

The ecological approach to perception is far from being dominant in the field of psychology; however, it has been receiving attention again with the emergence of the enactive and embodied views of the mind. ${ }^{3}$ These are all related theories that propose to construe cognition not as something that happens only inside our brains but as involving constitutively the interactions of the organism with its environment and as giving a fundamental role to its body in organizing and structuring the perceptible world. According to Gibson's approach, perception does not boil down to

3 For a more detailed discussion of the relation between the ecological approach and the enactive and embodied views of the mind, see Chemero (2009, pp. 17-44), Rowlands (2010, pp. 33-37), and Hutto and Myin (2017, second chapter). 
sensations, nor it can be taken as a mental process of interpreting or adding something to sensations. Indeed, sensations are incidental to perception, “detecting something can sometimes occur without the accompaniment of sense impressions” (Gibson 1968, p. 2). Perception, rather, is the direct pickup of environmental information for the control of behavior. It is not passive as sensations are; rather, it is the result of the employment of a system whose main function is to seek and extract information about the environment from the optical, mechanical, or chemical energy. Some of that information, as the size constancy of an object (about which I will say something later), is scattered in the environment and can only be picked up over time while the organism moves through its environment. Perception, therefore, must be construed as a system or, as I would prefer to say, a set of skills or abilities for the detection and discrimination of information available in the environment, skills whose employment normally takes time and requires the movement of the eyes, head, or body. The eye itself, for instance, is not the organ of vision; it "is part of a dual organ, one of a pair of mobile eyes, and they are set in a head that can turn, attached to a body that can move from place to place” (Gibson 2015, p. 47). All these elements working together for the purpose of picking up environmental information constitute a perceptual system.

The distinction between stimulation and stimulus information, which is closely related to the distinction above between defining the senses as channels of sensations or as perceptual systems, is significant for the ecological approach. Receptors are stimulated when they absorb an amount of energy above a certain threshold. Stimulation is a passive process. The traditional view of perception posits that punctate and momentary stimulation and the corresponding sensation comprise the basis of perception (Gibson 2015, p. 47). Because this sensory input "carries no information about its source in the world; that is, it does not specify its source,” (2015, p. 50) perception is understood as a creative process of embellishing the meager input of stimuli. Memories or background knowledge, whether learned or innate, must contribute to perception in some way. The ecological approach is 
founded upon a quite different assumption. When energy—such as mechanical, optical, or chemical —in space and/or in time is sufficiently structured to specify its source, then it carries information about its source. Information, for Gibson, "means information about, or specification of" (1968, p. 187). One thing specifies another if the former is univocally related to the latter by virtue of physical laws (Gibson 1968, p. 187). ${ }^{4}$ Now, if we assume that the basis of perception is not a momentary stimulus but a changing flow of stimulation, then the function of perception is to pick up information that is carried by the structured flow of stimulation and yielded by certain interactions between the organism and structured energy in its environment. To pick up such information is to directly perceive the source specified by that information; this process does not require the intervention of memories, representations, or inferences (Gibson 2015, p. 139; Michaels and Carello 1981, p. 2). This process is active since the organism needs to move and adjust its sense organs in order to have a structured flow of stimulation that carries ambient information. Perceptual systems control the flow of stimulation in order to resonate to ambient information.

To understand how energy can be structured, consider the case of optical energy. Each point of observation receives rays coming from different directions with different intensities depending on the surfaces that reflected them. Gibson calls a point like this one ambient light, because it comprehends all the rays of light coming from the surrounding ambient, and he contrasts this kind of light with radiant light, a singular ray of light that departs from a point, by reflection or by being an original source of light. Radiant light contains no information about its source, ${ }^{5}$ whereas ambient light

4 Gibson's notion of information as a relation of specification must not be confused with Shannon's concept of information (Gibson 2015, p. 232; 1968, p. 245). Whether the relation of specification depends necessarily on natural laws has long been debated. This claim seems to be defended by Gibson, as well as Turvey, Shaw, Reed and Mace (Turvey et al. 1981). Chemero, however, claims that strong regularities underpinned by conventions are enough (2009, pp. 116-120). See Heras-Escribano \& Pinedo (2016) for criticism of this position. More recently, Bruineberg, Chemero and Rietveld distinguish between lawful information and general ecological information. The latter is "any regularity in the ecological niche between aspects of the environment, $x$ and $y$, such that the occurrence of aspect $x$ makes the occurrence of aspect y likely” (Bruineberg et al. 2018, p. 7). In this paper I will utilize the definition of lawful information.

5 If this is the kind of light available to sight, then it is not surprising that it has been argued that distance is not given to us directly by vision but must be inferred. No ray of light contains information about how far it has traveled. See Smith's reconstruction of the Berkeley discussion about this point (Smith 2000, pp. 488-490). 
contains information about the reflecting surfaces due to the differences of intensity among the rays coming from different directions. If one surface in the ambient is changed, the ambient light in that environment changes as well, so the structure of ambient light specifies something in the environment (Gibson 2015, p. 45). ${ }^{6}$ Coal, for instance, modulates light in a particular way due to "its irregular, multifaceted, achromatic, and absorbent surface” (Michaels and Carello 1981, p. 25). Thus, the ambient light in an environment with coal is structured characteristically. That light is a structure that specifies coal and, therefore, carries environmental information about coal.

Energy patterns that are uniquely and invariantly tied to their sources in the environment carry information about the environment. The concept of invariant is crucial to understanding how an organism detects environmental information. For Gibson, the optic flux, as well as the mechanical, acoustic, and chemical fluxes, may reflect a lot of high-order information stored in the environment inasmuch as what is invariant, and therefore specify some lasting object or property, is revealed as such by changing what is inessential. ${ }^{7}$ In fact, “invariants are, quite simply, properties that tolerate certain transformations without changing” (Michaels and Carello 1981, p. 40). Thus, in many cases, "what is invariant does not emerge unequivocally except with a flux" (Gibson 2015, p. 66). A considerable amount of Gibson's work was dedicated to identifying the invariants stored in the environment. For example, in order to explain the perception of size constancy, traditional approaches assume that the perceptual system infers the real size of an object from its apparent size and its distance from the beholder. Those approaches must then explain how the information about the distance is initially obtained. Gibson's approach, however, requires no inferences. He claims that the information about the size constancy of an object is present in the environment, at least in an

6 These differences are necessary for the ambient light to contain information. In some situations, as when the environment is filled with dense fog, the ambient light is not structured, as at any point the rays coming from different directions have the same intensity. In this environment, no changes in a surrounding surface would have any impact on the ambient light. For Gibson, as nothing can be discriminated in this scenario with the help of the ambient light, this situation is a case in which sensation is not sufficient for perception (Gibson 2015, pp. 46-47).

7 Invariants that specify lasting entities are also referred to as structural invariants. However, there are also invariants for events-invariants that specify a style of change. These invariants are also referred to as transformational invariants. For a detailed discussion of these types of invariants, see Michaels and Carello (1981, p. 25-30). 
environment with a ground like ours, and can be directly picked up by a moving organism. He points out that in our environment "the rule of equal amounts of texture for equal amounts of terrain remains invariant” (Gibson 2015, p. 156); that is, an object close or far away from the perceiver occludes the same amount of terrain. The more distant an object is from the perceiver, the denser the texture of the terrain and of the object, but the relation between the object resting on the ground and the amount of ground texture that the former occludes remains the same. That invariant can be picked up by an organism while it approaches to or recedes from the object; as Gibson says, "the dimensions of things on the ground are perceived directly” (Gibson 2015, p. 160-161).

We are now in a better position to appreciate Gibson's view of perception as a system for discriminating or detecting things, properties, or events in the environment. As has already been pointed out, Gibson suggests a different starting point for thinking about the function of perception. What if, instead of the meager sensory input assumed by the traditional approach to perception, "the flux of stimulation at receptors does yield all the information anyone needs about the environment?” (Gibson and Gibson 1955, p. 34). Those systems may then be considered as discriminatory processes whose main function is to pick up the information available in such a flux. This is something the organism usually learns to do, although it may already be attuned to some specific environmental information through evolutionary processes (Michaels and Carello 1981, p. 79). An organism learns to perceive insofar as it learns to discriminate invariants present in its environment; perceptual learning, Jerome Gibson and Eleanor Gibson claim, “consists of responding to variables of physical stimulation not previously responded to" (Gibson and Gibson 1955, p. 34). Thus, they propose that the general function of the perceptual systems is to yield patterned specialized responses to specific invariants. These responses can be taken, I suggest, as specific discriminatory skills or abilities. As Gibson points out, “perceptual systems develop perceptual skills” (Gibson 1968, p. 51). ${ }^{8}$

8 It's worthwhile to mention that the ecological approach to perception is suitable to handle sensory substitution. This is because the aim of perceptual systems is to detect energy patterns. Informational structures, not sensations themselves, are meaningful. Thus, an optic pattern that is normally picked up by the visual system may also be 


\section{Discriminatory skills and environment}

The view that discriminatory skills can only be exercised in a proper environment still needs to be examined. Some of Gibson's comments may be developed to back up a conception of these skills as having a constitutive connection to the environment in which they were raised by an evolutionary or learning process. Because of how this connection is construed from an ecological approach, I submit that a discriminatory skill is exercised only in its proper environment; otherwise what we have is nothing more than a failed attempt to exercise that skill. Besides, there is no such thing as an unsuccessful exercise of a discriminatory skill. I will argue that there is a coherent and defensible sense in which it is right to say that If a discriminatory skill is exercised at all, then it is successful in picking up a particular. Three claims back up that proposal: (i) Invariants themselves are dependent on the environment; (ii) We pick up an invariant by perceiving affordances, which are relations between the organism and its environment; and (iii) Discriminatory skills are always a matter "of getting [dynamically] in closer touch with the environment” (Gibson and Gibson 1955, p. 34) over time. Let us unpack those claims one at a time.

\subsection{Invariants are environment-dependent}

It is important to emphasize that invariants are normally dependent on the environment; they specify features of an object or aspects of an event in a specific environment and cannot be understood in abstraction from that environment. The size constancy invariant, for instance, requires an environment with a ground-it does not even exist in the optic array in open air. ${ }^{9}$ Another example is the ability of sharks to electrically detect food, such as a flatfish. Edible organisms produce a bioelectric field that is "partially modulated in the rhythm of the living thing's respiratory

picked up by the haptic system provided that it is first converted into a haptic pattern. For further discussion on this point, see Favela et al. (2018).

9 Gibson reported that it can be very disorienting for an airplane pilot to fly through clouds if there is no way to see the ground. Even when there is some visibility, if the ground is out of sight, the pilot's acuity in perceiving size constancy is impaired (Gibson 2015, pp. xviii, 153). 
movements” (Turvey et al. 1981, p. 276). Thus, a shark can discriminate an edible thing by discriminating a certain type of bioelectric field. This is possible only because "in the niche of the shark 'an edible thing' and 'electric field of, say, type F' are nomically related” (Turvey et al. 1981, p. 277). The invariant that specifies edible things is dependent on a specific type of environment: the niche of the shark. This point has repercussions for how we should conceive discriminatory skills. It is to be expected that a discriminatory skill that has the function to pick up an invariant is able to do so at least in the environment that supports that invariant. An organism able to discriminate the size constancy of objects while it remains on the ground is not supposed to do so in situations in which the ground is not present or visible, because information on size constancy is not available in these situations. The main point here as to how discriminatory skills should be conceived is that, at least in environments in which an invariant is available, a discriminatory skill must be able to systematically pick up that invariant for it to be the case that it has the function to discriminate that invariant. Thus, there should be a systematic link between the exercise of a discriminatory skill in a certain type of environment and its success in picking up an invariant in that environment.

Such systematic links between the exercise of a discriminatory skill in a certain type of environment and its success in picking up an invariant is not enough to sustain that that discriminatory skill could not be exercised in another type of environment, even unsuccessfully. We must therefore unpack the second claim. The claim about the perception of an invariant through its affordances should be construed in the context of Gibson's understanding of perceptual systems for the control of behavior. ${ }^{10}$ According to Gibson, if we take seriously the idea that deliverances of perception should be useful for the control of behavior, then it is reasonable to assume the hypothesis that invariants are

10 This proposal should not be confused with the behaviorist idea that perception is reduced to a kind of performative behavior. An organism can perceive the size of a tomato before moving to a position in which the back of the tomato is put in view. Gibson's point is that the function of the perceptual systems-the pickup of invariants in the environment - is subordinate to the function of the performative and orienting systems (Gibson 2015, p. 234); that is, to the organism's control of its actions for achieving its needs and plans. However, at the same time, is true that some kinds of behavior serve perception. Adjustments of the eye, head, and body, movements, and probings are all encompassed by an exploratory system that is subordinate to perception (Gibson 1968, p. 57; Gibson 2015, p. 234). 
picked up or perceived in terms of affordances, that is, possibilities for action. As he puts it, "to perceive something is also to perceive how to approach it and what to do about it.” (Gibson 2015, p. 216).

\subsection{Affordances are relational in nature}

Affordances are resources in the environment that offer opportunities for behavior; for instance, a trunk knee-high above the ground offers to me the opportunity for sitting on it. An object with opposing sides that are less than a handspan apart affords grasping. These two examples illustrate an important point about affordances: they cannot be specified without reference to the organism. As Gibson says, "an affordance...points two ways, to the environment and to the observer” (Gibson 2015, p. 132). Affordances are relational in nature. In both cases, what the object affords depends on the features of the object. Whether an object is graspable depends on its width. Whether a surface can be sat upon depends on its height. At the same time, it depends on the organism as well. An object that is graspable by me does not necessarily afford grasping for a child; the same applies to objects that afford sitting on for me. Affordances are relative to possibilities for action, and it happens that I and a child have different sets of possibilities for action. However, there is no consensus among ecological psychologists regarding what in the organism underpins the possibilities for action. The options range from body properties (Warren 1984), to dispositional properties (Turvey 1992), to abilities (Chemero 2003). Here I will follow Chemero in claiming that affordances are relations between features of the environment and abilities of the organism. Thus, in order to perceive a trunk as affording the opportunity to sit on it, the organism must actually be able to sit on the trunk-it must have the proper ability to do so. That the body of an organism in virtue of its flexibility can be put in the right position of being seated is not enough. 
In this reading of Gibson's account of perception, abilities are important because they help the organism to track, explore, and pick up invariants. In fact, the perceptual system of an organism does not construct general and context-free representations of these invariants, but instead resonates to them (Gibson 1968, pp. 269-271) or is attuned to them (Gibson 2015, p. 238). Gibson uses that radio metaphor to call attention to the exploratory activity and continuous attunement of the organism to its environment in the process of picking up invariants. The emphasis on affordances also clarifies this point. For instance, imagine a very tired human being walking in a park. This person is approaching something that might be a trunk, but the invariants specifying its shape have not yet been picked up by the person's perceptual system, only part of them. The person then approaches the trunk in a way that discloses its height and shape. Because the person is tired, he or she is prepared to sit; that is, the person's ability to sit is ready to be exercised, the muscles of the back and legs are tensed in such a way that the person feels as if his or her body were soliciting him or her to sit on the trunk and rest. Besides, the person's ability partially shapes the way he or she approaches towards the trunk by helping the perceptual system track and explore the height and the shape of the trunk in relation to those parts of the body that are relevant to the action of sitting. If the person were engaged in a different activity — for instance, if he or she were running and trying to jump over trunks—then a different set of abilities would be in play; that trunk would be explored and approached in a different way, and its height and shape would be picked up in terms of other parts of the body. The affordance of sitting on and the affordance of jumping over refer to different relations between the trunk and the individual in question, because the possibilities for the action involved in each case are not the same. Which affordance is solicited and perceived in a given moment depends on the activity in which the organism is engaged and consequently on the abilities it brings to bear on the situation.

From the considerations above, two reasons can be given as to why a proper environment is necessary for the exercise of a discriminatory skill. First, if we perceive affordances, and affordances 
are relations between the environment and the organism, then the proper environment must be present for that relation to be perceived. A relation cannot exist without its relata. Second, the abilities of the organism that underpin its possibilities for actions are in play in an ongoing exploratory process by which the perceptual system of the organism tracks invariants in the surrounding environment. Insofar as these exploratory interactions make up part of the perceptual process, keeping the organism in contact with its surrounding environment, it can be said that perception cannot occur unless the organism finds itself in the proper environment to pick up the invariant that has been just explored.

It seems that once we have accepted Gibson's active account of perception, it is difficult not to acknowledge the fundamental role that the environment plays in perception. However, the two reasons given above for taking a proper environment as necessary for exercising discriminatory skills could be overridden by the following objections. First, even though affordances are relations between the environment and the organism and they cannot exist without their relata, it does not follow from that that affordances are perceived whenever the perceptual system finishes a process of perception. If it is possible to "perceive” an illusory affordance, then it would be possible to exercise a discriminatory skill without being in the proper environment. Second, even though the exploratory activity of a perceptual system keeps the organism attuned to its environment, nothing of what has been said about this activity implies that it could not be bypassed by the perceptual system, yielding a perception without the help of that exploratory activity. Again, one could "perceive” an illusory affordance, and the same consequence as before follows.

\subsection{The radio metaphor and perception as the act of picking up information}

In order to respond to these worries, it may be stressed that Gibson's approach is committed to a stronger claim, namely, that exploratory activity is not only a possible part of the perceptual process 
but a necessary and constitutive one as well. Once we have a proper understanding of the role that the third claim (iii) plays in his account of perception, we can dismiss the objections above. The third claim is necessary to connect Gibson's characterization of the general function of perceptual system as “a discriminative process” (Gibson and Gibson 1955, p. 34) with his characterization of a particular act of perceiving as "the act of picking up information” (Gibson 2015, p. 229). In other words, we need to understand how an organism acquires discriminatory skills through which it can pick up invariants.

Discrimination is not to be understood as a process of singling something out based on sensations; instead, it is based on affordances - that is, an object is discriminated from others based on the possibilities for action it offers from the perspective occupied by the organism. Exploratory activities and "adjustments of the perceptual organs” (Gibson 1967, p. 163) are not optional; they constitute discrimination. The radio metaphor is again relevant. These activities keep the organism attuned to an object while disclosing its affordances. Over time, they are necessary and sufficient for bringing about invariants in the flow of stimulation. Thus, the third claim establishes a constitutive connection between exploratory activities and discriminatory skills. As Gibson claims, "the process of pickup involves ... overt movements that can be measured, such as orienting, exploring, and adjusting” (2015, p. 251). These activities in turn cannot be understood independently from the environment in which they occur. They are always doings from a place to keep the organism in touch with some environmental structure; the adjustments therefore depend on the organism and the environment, which constrains how the organism can approach a surrounding object. After a routine of orienting and exploratory adjustments of the perceptual organs is established in relation to a environmental invariant, in a way in which responses of the organism to that particular invariant can be said to resonate to it, the organism is in the possession of a discriminatory skill or ability. ${ }^{11}$ Learning a

11 A history of interactions with an object or property is essential for the acquisition of a perceptual or discriminatory skill. By those interactions an organism "can have found out that this or that action was actually afforded in the past, and this can be the basis on which its current perception of its environment can have become sensitive to these 
discriminatory skill is a process of attunement through which the organism adapts to its environment, giving rise to an organism-environment system. One consequence of this process is the great degree of symmetry that arises between organismal states and environmental states (Shaw and McIntyre 1974, p. 278). For instance, through the orienting and adjusting activities that constitute a discriminatory skill, the structure of an environmental invariant is reflected in a symmetrical flux of stimulation of the organism attuned to that invariant. The reason, then, why a discriminatory skill can be exercised only in its proper environment is very simple. Since its mechanism for picking up information is a resonating one, which involves modulating the flux of stimuli to reflect or resonate to an environmental invariant, this invariant must be present for the continuous adjustments of the sense organs and body through which a discriminatory skill controls the flux of stimuli in order to fulfill its aim. Motor feedback — the presence or absence of impediments-is then crucial for that control. In a nutshell, to resonate to its environment, the organism must be in that environment. Similarly, perception, the act of picking up information, is never an instantaneous act but a continuous act “of getting in closer touch with the environment” (Gibson and Gibson 1955, p. 34).

It may be objected that we have modeled discriminatory skills in the light of a resonating mechanism because we assumed that perception is the act of picking up information. A weaker assumption-that perception is the act of picking up information in general-would lead us in another direction. However, it's clear to Gibson that the ecological approach to perception involves a redefinition of perception (Gibson 2015, p. 228). That he has intended to define perception along these lines, making the strong claim that to perceive is always the act of picking up an invariant, is clearly expressed in his acknowledgment that his theory of perception cannot be used to "account for hallucination or delusions or, in fact, for any kind of maladjustment” (Gibson and Gibson 1955, p. 34). These phenomena are no less important for understanding the behavior of an organism, but they

environmental properties.” (Myin 2016, p. 99) Gibson also claims that "the pickup of information reinforces the exploratory adjustments of the organs that make it possible” (1968, p. 271). 
must be explained by "supplementary assumptions," that is, "a theory of perception should certainly allow for misperception, but it can hardily at the same time be a theory of misperception” (Gibson 1968, p. 287). Misperception can occur because the information available is inadequate-it is not sufficient for specifying an environmental invariant_or even when it is adequate, because it is not picked up (Gisbon 2015, p. 233). In either case the definition of perception is not satisfied. Let us examine each of these cases.

Take the case of the Müller-Lyer illusion. It consists of two parallel lines, one of which ends in inward pointing arrows, the other which ends with outward pointing arrows. The latter line appears to be bigger than the former. This case is supposed to be an illusion because the lines have the same length, whereas we have an experience of the lines as having different lengths. However, we are assuming that one line isolated from other cues constitutes information for the length of the line. This assumption is rejected by Gibson: He points out that the "stimulus information for the length of a line is altered by combining it with other lines” (Gibson 1968, p. 303). Given the environment in which we live, the first line contains partial information for the ridge of a roof seen from below, while the second line contains partial information for the ridge of a roof seen from above. Thus, given the information conveyed by these lines in normal situations, it is not surprising that one line appears to be bigger than the other. In fact, this is reason for not treating this figure as a paradigmatic case of illusion. If our criterion of reality is not the length of the line in the retinal image, as it was assumed by the traditional psychologist (Michaels \& Carello 1981, p. 91), but the information conveyed by the combinations of lines, then, according to projective geometry, the lines appear as they should. In any case, this is an episode of misperception, because it is a case of picking up a partial information for an invariant instead of an invariant itself. Notice that the Müller-Lyer drawing cannot be explored and focused from different perspectives in the same way in which the lines composing the shape of the ridge could be explored and focused by a moving organism. Any 
invariant related to the length of the ridge that could be picked up by a moving organism is not available in the Müller-Lyer drawing: Partial information is being mistaken for the complete information about an invariant. It is not a case of perception, since perception is the act of picking up invariants instead of ambiguous or incomplete information. In fact, many so-called optical illusions are cases of picking up incomplete information. In this respect, it is interesting to notice that in some situations of insufficient information, it is not uncommon that the perceptual system keeps trying to reach additional information by launching more exploratory activities and adjustments of the sense organs (Gibson 1968, p. 303).

There are other situations in which an invariant is present in the environment, but the organism may be unable to pick it up. This can happen for many reasons, by failure of organ adjustment at high intensity stimulation, physiological after-effects, over-selective attention, after-effects habituation, etc. (Gibson 1968, pp. 304-310). For instance, at very high levels of illumination, such as when one is looking directly at the sun, the intensity of the light is too great to be registered by the human eye's photoreceptors. Invariants specifying a flying bird can be present but may not be picked up in this situation. Another case of deficiency of the perceptual process is that of afterimages, which are due to the way the retinal photoreceptors work. After being over-stimulated for a long period of time by uninterrupted light, photoreceptors adapt to the stimulation by losing sensibility. Then, for a brief period after the interruption of stimulation, the photoreceptors continue to be excited, yielding a sensation of a patch of color in whatever direction one looks. Afterimages may distract attention and affect the process of picking up invariants, but normally they do not impede that process, requiring more effort from the organism to compensate their distracting effect (Gibson 1968, p. 306).

It may be argued that Gibson is making an arbitrary move by defining perception in the way he does. The nature of perception, the argument goes, rather than being decided by definition, is something 
we should discover by doing experiments. However, what must be assessed empirically is Gibson’s theory as a whole, not particular bits of it. Furthermore, his procedure is a very common one in every scientific corner, as is well illustrated by Duhem's discussion about the definition of free fall. Free fall is defined as uniformly accelerated motion, wherein acceleration is constant and directed to the center of the Earth. If we observe the fall of a heavy object and notice that this object does not fall in accordance with that definition, we should not then dismiss the definition, but instead conclude that the object does not fall freely. In this situation, it must be assumed that some force, which then should be investigated and classified, have intervened to prevent the object from falling freely. Definitions that are fundamental for a scientific theory "cannot be contradicted by any experiment" (Duhem 1991, p. 208); rather, they set up the framework of a research program. A definition and the whole scientific framework revolving around it may be discarded only if the set of different causes and forces assumed to explain diversions from the definition starts to become much too complex and to generate theoretical difficulties rather than solutions and explanations. In Kuhnian terms, this may be the moment for a paradigm shift or scientific revolution (Kuhn 2012). Similar considerations apply to Gibson's approach to perception. If we observe an organism trying to pick up an invariant and notice that it fails to do so, or if we observe that an organism behaves as if there were an invariant that is not present in the surrounding environment, we should not dismiss Gibson's definition of perception, but instead conclude that these situations involve cases of misperception, that some cause must have intervened to prevent the organism from fully exercising a discriminatory skill. Insofar as Gibson's whole theoretical framework shows itself to be fruitful, there is no reason to reject his definition.

\section{Disjunctivism and ecological psychology}

Disjunctivism can be characterized by the rejection of the following assumption that, following McDowell (1982, p. 471), can be called the highest common factor assumption: 
Subjects who on seeing X/an F/something $\mathrm{G}$ have an experience such that it looks to them just as if $\mathrm{X} / \mathrm{an} \mathrm{F} /$ something $\mathrm{G}$ is there could have the very same experience if they were merely hallucinating X/an F/something G. (Millar 2016, p. 177)

Thus, while the traditional view claims that seeing an $\mathrm{X}$ and hallucinating an $\mathrm{X}$ involve the same type of experience, which could be described by a looks statement such as "It looks to S as if there is an X," the disjunctivist view sustains that these episodes involve different types of experience at a fundamental level, and that that look claim should rather be analyzed as stating a disjunction such as "S sees an $\mathrm{X}$ or she is having the hallucination of an $\mathrm{X."}{ }^{12}$ This does not mean that according to disjunctivism those experiences could not have anything in common. Even if they did, this would not turn them in experiences of the same type, at least not at a relevant explanatory level.

To avoid misunderstandings, it is important to clarify the connection between the ordinary usage of perceptual verbs such as "see”, "hear”, and "perceive” and what is claimed by disjunctivism. It is often acknowledged that perceptual verbs are primarily verbs of success or, as Ryle puts it, “verbs part of the business of which is to declare a terminus” (1954, p. 102-3). Perceptual verbs indicate the successful terminus of a process of detection or discrimination; for example, the verb "win" in the sentence "John won the race" indicates that John was successful in reaching the end of the race first. Analogously, the sentence "John sees that bird” does not indicate that John's eyes are directed toward a place where a bird can be found but that John has successfully detected or discriminated the bird from its surrounding. According to this usage, success verbs do not describe processes, they manifest an achievement. I can, in fact, "be looking for or looking at something, but I cannot be

12 John Hinton was probably the first philosopher to explicitly propose this kind of analysis for looks claims (Hinton 1967) and to suggest that we are not obliged to accept that there is a common element, a shared inner experience, to perceptual and illusory experiences (1967, p. 223). As to whether naïve realists, who had also endorsed before Hinton that there are two types of experience in the appropriate sense, should count as disjunctivists or not is an open issue (Snowdon 2008. p. 40). 
seeing it. At any given moment either I have not yet seen it or I have now seen it” (Ryle 1954, p. 103). Of course, perceptual verbs can also be used in situations that do not involve successful detection or discrimination. We commonly use these verbs to describe dreams or episodes of hallucination; this use is not inherently problematic, provided we keep in mind that these are not cases of successful detection or discrimination. In these occasions, we are just describing how things look or appear to us. Let us call this usage of perceptual verbs the "non-success usage” and the former one the "success usage". Thus, it is correct to say that the disjunction "S sees an $\mathrm{X}$ or she is having the hallucination of an X" captures, in a more perspicuous way, the ordinary distinction between the success and non-success usage of perceptual verbs. It is not a novelty that disjunctivists tend to present their view as articulating common-sense ideas (Pritchard 2015, p. 6; McDowell 1996, p. 113; Millar 2016, p. 63). However, it would be wrong to conclude that disjunctivism is nothing more than the ratification of that distinction. For one thing, non-disjunctivists are also perfectly comfortable with that distinction. The point at which disjunctivists and their opponents disagree is how to explain this distinction. The latter group posits that the difference between the success and non-success usage of perceptual verbs has nothing to do with agent's experiences. ${ }^{13}$ The presence, in a successful case, and the absence, in a non-successful case, of the object seen is completely extrinsic to the agent's experiences. Thus, the assumption of the highest common factor is open to them. For the former, on the contrary, the difference is intrinsic to experience. In a successful case, our experience involves the object present, in fact, one sees the object present because one’s perceptual experience discloses it, whereas, in a non-successful case, our experience does not involve any environmental object. Therefore, an episode of perception and the corresponding episode of hallucination are fundamentally different types of experience. Perceptual experience is world-involving, according to the disjunctivist.

13 This case is usually referred to as metaphysical disjunctivism; disjunctivism about the nature of experience. Others prefer to explain the difference between the successful and non-successful usage of perceptual verbs in relation to the epistemic warrant that experience can provide, which is usually referred to as epistemic disjunctivism. For an introduction to varieties of disjunctivism, see Haddock and Macpherson (2008). 
Gibson's view of perception is in agreement with the rejection of the highest common factor assumption:

There can be direct or immediate awareness of objects and events when the perceptual systems resonate so as to pick up information and there can be a kind of direct or immediate awareness of the psychological states of our sense organs when the sensory nerves as such are excited. But these two kinds of experience should not be confused, for they are at opposite poles, objective and subjective. Only the former should be called perceptual experience. (Gibson 1967, p. 168)

As was shown in the last section, according to ecological psychology there is a fundamental asymmetry between the exercise of a discriminatory skill, which yields a case of perception, and the failure to exercise that skill, which yields a case of misperception. There is no highest common factor being shared by these cases. In a case of perception, the organism picks up an invariant and is aware of something in its surrounding environment (Gibson 2015, p. 244), whereas in a case of misperception either no invariant is picked up or an insufficient information is picked up; in both cases the organism is not aware of something in its surrounding environment, even though it may have all sorts of sensations. Thus, cases of perception and cases of misperception are different, and this difference is warrant enough to motivate disjunctivism about the nature of experience. Further, as picking up information is to perceive directly the source of that information, perception is also world-involving according to the ecological approach.

A common theme amongst those who articulate a version of disjunctivism is that the exercise of our perceptual skills or abilities should not be construed as mere reactions to objects in the environment, nor simply as "a matter of my being affected in certain ways as a result of the presence” (Millar 2016, p. 191) of certain objects in the environment. On the contrary, the exercise of a perceptual 
skill should be construed as active, as something an agent does (Noë 2004, p. 1) and therefore as something that can be done intelligently. This is because perceptual skills are, as claimed before, skills acquired by learning and attributable to agents. It's the organism as a whole who learns to discriminate. These skills have the function of “enabling access to what there is” (Noë 2015, p. 2), of providing “openness to the world” (McDowell 1996, p. 111) or of putting us "in cognitive contact with objects or facts” (Millar 2016, p. 194). Thus, the exercise of a perceptual skill or ability, by putting us in cognitive contact with an object or by providing responsive access to its presence, results in the acquisition of knowledge. When something goes wrong, maybe because one finds oneself in an inadequate environment or maybe because some enabling condition is not present, despite the attempt to exercise a perceptual skill, none is actually exercised; in this case no perceptual knowledge is acquired. This view explains well why perceptual experience and hallucinatory experience have different natures. The former is generated by the exercise of a perceptual skill which successfully puts the agent in cognitive contact with some object or event in her environment, the resulting perceptual experience involves the awareness of that object or event, whereas the latter is a failed attempt to establish that contact, the agent is aware of no object in her environment in virtue of her hallucinatory experience.

This description of perceptual abilities as acquired skills fits well with Gibson's view of perception. I’ve already quoted Gibson’s claim that “perceptual systems develop perceptual skills” (Gibson 1968, p. 51) by a process of attunement to an invariant. In fact, “a perceptual system is altered when it is attuned to information of a certain sort. The system has become sensitized. Differences are noticed that were previously not noticed” (Gibson 2015, p. 243). Becoming attuned to information is the same as acquiring a skill of access to the object or event specified by that information. In line with the above construal of perceptual skills, "the theory of information pickup makes a clear-cut separation between perception and fantasy, but it closes the supposed gap between perception and 
knowledge” (Gibson 2105, p. 246). Thus, there is a perfect match between the ecological view of perception and the disjunctivist construal of perceptual skills.

In the previous section, I claimed that, according to the ecological view of perception, perceptual skills can only be exercised in a proper environment. One may wonder whether disjunctivism supports this claim. On this matter, there is no consensus. McDowell, for example, accepts that there may be defective exercises of perceptual abilities (2011, p. 38). According to him, fallibility should be applied to abilities or capacities, "there can be exercises of the capacity in which its possessor does not do what the capacity is specified as a capacity to do” (2011, p. 37). Alan Millar thinks differently; fallibility should be applied to the attempts at exercising an ability. According to him, the manifestation of a skill or an ability is a notion of success (Millar 2009, p. 224), the manifestation is the subject's fulfilling the function of that ability. One may be said to have attempted to exercise an ability, but if the ability's function has not been fulfilled, one did not exercise it. He contends that this construal of perceptual skills is necessary to explain how cognitive contact is achievable. If one assumes that perceptual skills provide cognitive contact with surrounding objects, then the skills "in question are individuated in such a way that they do not count as being exercised unless knowledge is acquired” (Millar 2007, p. 194). ${ }^{14}$ The details of this transcendental argument about what is required to secure cognitive contact are not important here. The relevant point is the match between the ecological and Millar's disjunctivist construal of perceptual skills. On one hand, this increases the validity of examining the epistemological consequences of the ecological view of perception in

14 It may be objected that some performances are so difficult that even someone highly skilled in completing the performance will not succeed every time she tries to exercise that skill. This is the case, for instance, of chicken sexing. Even the most skilled in this task will, at best, classify 1,000 chicks per hour with $98 \%$ accuracy (Biederman and Shiffrar 1987, p. 640). This seems to challenge the assumption that all exercises of a skill must be successful. It would be odd to say that these people only exercise the skill $98 \%$ of the time but fail to exercise it in the other $2 \%$ of the time. In cases such as this one, we require, as Millar points out, the notion of a success-rate ability (2016, p. 70). A chicken sexer who has the ability to classify 1,000 chicks per hour with $98 \%$ accuracy will succeed in classifying with $98 \%$ accuracy every, or even nearly every, time she exercises her success-rate ability during the course of a 1,000-chick sequence. Again, abilities or skills are individuated in such a way to preserve the connection between exercise and success. 
the light of disjunctivism. On the other hand, Millar's version of disjunctivism may be backed up by a scientific view of perception.

What is the kind of knowledge acquired by the exercise of a perceptual skill? As perceptual abilities are developed or acquired skills, provided that the function of putting us in cognitive contact with objects or facts is fulfilled, there is a wide range of possibilities: From practical knowledge to objectual knowledge to propositional knowledge. In line with the ecological view of perception, I submit that perceptual or discriminatory skills provide primarily practical knowledge. ${ }^{15}$ The knowledge obtained by the exercise of perceptual skills is knowledge of affordances, that is, knowledge of how to deal with surrounding objects and events to satisfy the organism's practical needs. The possession of discriminatory skills opens up to the organism what may be called a space of possibilities for actions, in allusion to Sellars' logical space of reasons (Sellars 1991). Discriminatory skills carry a particular type of intelligibility of the world, namely, a practical mode of understanding (Noë 2015, p. 3). ${ }^{16}$ In this sense, to have perceptual knowledge of an object is not only to be aware of that object but mainly, depending on the task, to be aware of some of its possibilities for action and to be prepared to enact them. All that is necessary for an organism to possess practical knowledge regarding an object in its environment is that the organism is able to keep its cognitive contact with that object through its discriminatory skills, which, remember,

15 It's perfectly doable to conceive, as McDowell does (1996, 2011), perceptual skills as involving conceptual resources from the beginning and then to sustain that the exercise of these conceptual-perceptual skills provides propositional knowledge. This is not the path I'm following, at least if it is assumed that concepts are contextindependent entities that satisfy Evans' generality constraint (EVANS 1982, p. 104) and structure propositional thought. As is strongly suggested by ecological psychology, non-conceptual perceptual skills can be acquired much before we are able to acquire conceptual-perceptual skills, and this is something that we share with animals. The ecological approach, as Turvey et al. emphasize, "is concerned with the perceiving that goes with acting" (TURVEY et al. 1981, p. 240) or with how perception serves to guide and control action, whereas the traditional approach to perception is concerned with the fixation of perceptual beliefs. Gibson is focused on low-level phenomena. Besides, as Dreyfus suggests, we should try to explain how the "upper stories of the edifice of knowledge" (2006, p. 48) — such as perceptual beliefs—rest upon the non-linguistic coping going on on the ground floor. This requires first a good understanding of what is going on on the ground floor.

16 In the view presented here, objects are not purely given to us in perception, they are intelligibly apprehended and structured in the space of possibilities for action. Perception, as we have been pointing, is not passive but active, it involves the employment of agent's discriminatory skills. Thus, Sellars' charge that a pure given cannot be an episode of knowledge (Sellars 1991, pp. 127-134) does not apply to the view presented here. 
involve exploratory activities. Perception, then, is something we do (Myin 2016, p. 99). To see an apple, for instance, is to perceive its affordances, is to know how to keep it in view, to reach it, to manipulate it, among other things. I call this view of perceptual skills or abilities ecological disjunctivism.

\section{Ecological disjunctivism and the distinguishability problem}

If it's agreed that a case of perception and its corresponding case of hallucination are subjectively indistinguishable (McDowell 1996, p. 112) (Pritchard 2012, p. 20), then, even if these cases represent different kinds of experience, as disjunctivism claims, it may be argued that one can never know whether one is perceiving or not. If the basis for distinguishing one case from the other is by introspecting their phenomenal qualities, then it seems there is no way to distinguish between a case of perception and its corresponding case of hallucination. As the argument goes, if one cannot distinguish one case from the other, then one cannot know one is perceiving and, therefore, obtain knowledge about the world through perception. This is the distinguishability problem. The openness to the world promised by disjunctivism would not be enough to secure knowledge of the world.

First, notice that we are traditionally invited to imagine a perceiver in a stationary situation looking at a particular object and then to conceive the possibility of that perceiver having the same experience in a situation of hallucination. If we assume the snapshot view of perception, it is not a surprise that we must allow the possibility and even the existence of many pairs of indistinguishable episodes of perception and hallucination. However, if we assume Gibson's view of perception and understand that perception occurs over time through the exercise of discriminatory skills that usually require movements of the eyes, head, and body to pick up invariants in the environment, it is then disputable whether we need to allow the existence and even the possibility of these pairs of

cases. To know whether you are looking at a barn facade or an actual barn, you need just to 
approach it laterally and pick up the invariant for a flat surface or the invariant for the depth of a barn. That you in a stationary position are unable to tell one from the other if they are centered in your visual field is not an indication of any discriminatory limitation of your perceptual system. Discriminatory skills are not supposed to be exercised without requiring some sort of exploratory activity. Besides, picking up the invariant for a flat surface is different from picking up the invariant for the depth of a barn; the exploratory activities involved in these acts of perception are not the same.

But then it may be argued that the same flux of stimulation that is obtained by exploring a real barn could also be obtained by stimulating our sense organs over time in a similar manner, yielding then a corresponding case of hallucination. Hallucination would happen over time as much as perception. This worry, however, ignores the constitutive connection between discriminatory skills and exploratory activities. The perceptual system is not passive in relation to the flux of stimulation, it's active in exploring and generating it. The process of picking up an invariant in the flux of stimulation, the exercise of a discriminatory skill, depends on the fact that this flux is under the control of the organism in the sense that the flux changes over time due to exploratory activities of the organism. The perceptual systems is not insensitive to feedback input from the motor system. To see the importance of this point, let's take the case of afterimages first. They cannot be "explorable, or investigable or susceptible to increased clarity by sense-organ adjustments” (Gibson 1970, p. 426), there is no way to approach to or recede from or focus on an afterimage. What cannot be explored by the adjustments of sense-organs cannot be perceived. Thus, afterimages cannot be the object of an episode of perception. We can, of course, have an afterimage of a star in the sense that we have sensations that are similar in some respects to the experience of seeing a star, but we cannot, according to the ecological approach to perception, perceive them. As Gibson points out, the 
brain "alone can generate experience of a sort. What it cannot do is to generate perceptual experience” (Gibson 1970, p. 426).

Gibson devised a test of reality for distinguishing cases of perception from cases of imagining, dreaming, or hallucination based on the connection between discriminatory skills and exploratory activities. His criterion is as follows:

Whenever adjustment of the perceptual organs yields a corresponding change of stimulation there exists an external source of stimulation and one is perceiving. Whenever adjustment of the perceptual organs yields no corresponding change of stimulation there exists no external source of stimulation and one is imagining, dreaming or hallucinating. (Gibson 1970, p. 426)

The motor input is, thus, crucial for the perceptual system. Let's take the barn case again. The perceptual system distinguishes the visual flux of stimulation caused by the real barn from the visual flux of stimulation caused by some neurological disturbance based on motor information. While exploring a barn, the organism needs to adjust its perceptual organs in order to maintain the barn in focus or to obtain a clearer view of it. These adjustments are followed by changes in the flux of stimulation. In a similar way, reversing movements yields a corresponding change of stimulation. For instance, moving toward the barn can be reversed by moving away from it. The stimulus flux reverses correspondingly. Besides this causal relation between adjustments performed by the organism and changes in the stimulation, another criteria for reality "is whether you can discover new features and details by the act of scrutiny” (Gibson 2015, p. 245). I can discover a hole in one of the walls of the barn by inspecting it while I get closer to it. Notice that a causal relation is also implied in this case. A new "property" may pop up in an hallucinatory experience but not as an outcome of activities of scrutiny. Thus, the flux of stimulation that is obtained by exploring an environmental object, like a real barn, is not the same as when one is having a "corresponding” 
hallucination. ${ }^{17}$ The former is a controlled flux but not the latter. ${ }^{18}$ This distinction is made by the perceptual system itself; as is suggested by Gibson, "reliable and automatic tests for reality are involved in the working of a perceptual system.” (Gibson 2015, p. 245) ${ }^{19}$

However, it may be argued that the distinguishability problem poses a question that must be answered in a first-person perspective. The question is whether an agent, not its perceptual system, distinguishes between perception and hallucination. The claim of pairs of indistinguishable cases of perception and hallucination is meant to be understood in the specific situation in which the organism (the agent) is confined to its own sensations available at the present moment and then is asked to tell those cases apart. In response to that challenge, the first thing to point out is that even if it were granted that the task of distinguishing between cases of perception from cases of hallucinations should be addressed by appeal to sensations only, it would not follow that an organism unable to accomplish this task on that basis cannot have perceptual knowledge of any sort. This is because, in the view presented here, the exercise of discriminatory skills, which can only happen in the proper environment, is sufficient to yield perceptual knowledge. No intellectualist

17 Beaton, relying on the sensorimotor theory of perception, advanced a very similar approach to how perceptions are distinguished from hallucinations and illusions. According to him, we should look at the similarities and differences between "actions an agent would take (if appropriately tested) when imagining (or hallucinating or having an illusion of or dreaming about) a given object (or property, etc.) and when actually perceiving such an object.” (2016, p. 271) This is very close to Gibson's point outlined above about the different effects of adjusting the sense organs when perceiving and when imagining, dreaming or hallucinating.

18 In a classical study with newborn kittens, Held and Hein (1963) gathered evidence on the importance of motor feedback for acquiring spatial notions. In their experiment, a group of kittens were allowed to move around a cylinder with vertical strips. A second group of kittens were exposed to the same stimuli, not by their own movements, but by a device that moved them around the cylinder. By mechanical linkages to the gondola attached to the active kittens, this device was able to mimic the movements of these kittens (1963, p. 873). Thus, both groups were exposed to the same visual stimulus flux. However, only kittens from the first group learned to navigate and move in the setting, while the kittens from the second group remained incapable to orientate in space.

19 These considerations might not be sufficient to deal with the case of a virtual reality that is controlled by one's movements. I am grateful to one of the anonymous reviewers for having pointed this out. This is a topic that raises many issues and would require much more space than I have to address them. However, I will make two comments about this case. First, many existing systems of virtual reality produce experiences that are distinguishable from our normal perceptions. This is because these systems are not so responsive to movements as our perceptual system is. Second, in the limiting case where a virtual reality system would produce over time a virtual reality that resembles our non-virtual reality, including the relations between movements and changes in experience, I would be inclined to claim that an agent equipped with the proper skills can perceive in this virtual reality, and that this reality is real after all. I would have then to commit to a view of reality that comes in degree, having our non-virtual reality as the limiting case, but this is a discussion for another paper. For a response to this objection from a sensorimotor approach, see Noë (2004, p. 224-225). 
condition, such as being able to place a perceptual episode in the space of reasons, is necessary for perceptual knowledge. If the distinguishability problem makes this assumption about knowledge, namely, that an episode can be a state of knowledge only if the agent is able to place it in the space of reasons, then so much worse for the problem.

Nevertheless, we may consider what it would look like for an agent to have second-order knowledge, more specifically introspective knowledge of one's experiential states, in the approach developed here. The second point, then, is that introspection, although it is a system distinct from perception, should be conceived as a skill as well—say, the skill for distinguishing episodes of perception from episodes of imagination, dreaming, and hallucination—and as such it can only be exercised in a proper environment and under certain conditions. At first glance, there is no reason why we should restrict the input for such skill or ability to sensations available at the present moment. Taking into consideration Gibson's test for reality, information about the adjustments of the perceptual organs delivered by proprioception may also be accessible to the agent's introspection. It may be the case that we need to learn to introspect as much as we need to learn to perceive and that introspection cannot be exercised in some situations, such as when the organism is dreaming, because the information necessary for exercising introspection is not available to the organism. Eventually, we may have to conclude that introspection is best conceived disjunctively in the same way as perception. In that case, it would be impossible to distinguish episodes of perception from other types of episode in some situations, but this is no barrier to the possibility of introspective knowledge in the case that the environment and the conditions are good for the exercise of such introspective skill, such as when the organism is awake and has access to information delivered by proprioception. In the proper conditions and environment, the exercise of this skill would yield introspective knowledge of the type of the episode one is having. The fact that this skill does not operate only upon sensations does not preclude it from yielding knowledge in the 
first-person perspective. It is not the aim of this paper to spell out the details of how such ability would work; for our purposes, it is enough to outline its general shape.

According to the view of skills as environment-dependent, there is no reason to grant that there can be pairs of indistinguishable episodes of perception and hallucination, even if we limit ourselves to the first-person perspective. On the contrary, as these episodes differ in nature, we have reason to think that it is perfectly possible for an organism to have or acquire a skill that explores that difference in order to tell those episodes apart in the first-person perspective. Thus, the distinguishability problem can easily be addressed by an organism with the appropriate skills. The problem seems to be a hard one only if we think that in the first-person perspective the organism has access to nothing more than its present sensations. However, why should we limit in that way the resources available to the organism in the first-person perspective? We should, rather, accept deliverances from any first-person skill ${ }^{20}$ working in proper conditions as legitimate starting points upon which new skills can rest.

Assuming the possibility of such introspective skill, what the organism gains by exercising this introspective ability is knowledge of affordances of its own mental episodes. In a recent paper, Tom McClelland spelled out the workings of such introspective skill (McClelland 2015) in more detail, extending the notion of affordances for that purpose. Although Gibson introduced the notion of affordance as mainly applicable to environmental objects and events, there is no reason for not allowing it to encompass affordances of mental states as well; that is, the possibilities for actions that mental states offer. These possibilities for action do not necessarily require bodily movements — they can be in a broad sense actions of a mental sort. Typical mental actions comprise attending,

20 This raises the issue of what constitutes a first-person skill. Following Lynne Rudder Baker, we can say that a firstperson skill or ability must exhibit the marks of consciousness and intentionality or self-consciousness. As to the first disjunct, which interests us here, the skill must be one by which the organism interacts "consciously and intentionally with the environment” (Baker 2013, p. 172). As we have been arguing, consciousness and intentionality are not instantiated only by awareness of sensations. Perceptual discriminatory skills exhibit equally the marks of consciousness and intentionality by making us aware of environmental invariants. 
expecting, evaluating, deciding, calculating, imagining, and judging. A perceptual state, for instance, in some situations, may afford a decision to perform an action, whereas a hallucination may afford a decision to not perform that action. The details do not matter here; the main point is that the introspective skill outlined above, in the proper environment and conditions, can make it possible to distinguish perceptual episodes from hallucinatory episodes, offering the organism a richer repertory of possibilities for action. Thus, in the view developed here, one can have perceptual knowledge of the affordances of an object and introspective knowledge of the affordances of that episode of perceptual knowledge. I submit that it is an interesting consequence of the way skills and abilities were suggested to be construed here that there may be second-order practical knowledge. It would be interesting to investigate what this view of discriminatory skills indicates about the difference between the perception of affordances of a particular in opposition to affordances of a type of particular - that is, how the view deals with perceptual particularity (Schellenberg 2016).

\section{Concluding Remarks}

According to the view developed in this paper, discriminatory skills can be exercised only in their proper environment. We can understand this conclusion by construing discriminatory skills through the radio metaphor. Discrimination is a resonating process through which an organism's perceptual systems become attuned to invariants. The exercise of perceptual skills gives us perceptual knowledge of affordances, that is, knowledge of how to deal with surrounding objects and events for the satisfaction of our needs and projects. The failure to exercise a discriminatory skill, which is same as the failure to pick up information, yields an episode of misperception. Thus, perception and misperception are different in nature. The ecological and disjunctivist views of perception are wellsuited to one another. Disjunctivism benefits from the scientific credentials of ecological psychology, while ecological psychology benefits from the epistemological framework provided by 
disjunctivism. One consequence of the proposed way of construing perceptual skills is that the distinguishability problem can be easily addressed. Even a lasting hallucinatory experience is distinguishable from a corresponding perceptual experience because the former does not result from a controlled flux of stimulation, whereas the latter does. This motor difference may also be the basis for an introspective skill to discriminate between affordances of episodes of perception and affordances of episodes of hallucination.

Acknowledgments I would like to thank the two anonymous reviewers for their valuable suggestions and critical feedback on this manuscript.

\section{References}

Baker, L. R. (2013). Naturalism and the first-person perspective. Oxford: Oxford University Press.

Beaton, M. (2016). Sensorimotor direct realism: How we enact our world. Constructivist Foundations, 11(2), 265-276.

Biederman, I., \& Shiffrar, M. (1987). Sexing day-old chicks: A case study and expert system analysis of a difficult perceptual-Learning task. Journal of Experimental Psychology: Learning, Memory and Cognition, 13(4), 640-645.

Bruineberg, J., Chemero, A., \& Rietveld, E. (2018). General ecological information supports engagement with affordances for 'higher' cognition. Synthese, https://doi.org/10.1007/s11229-018-1716-9.

Burge, T. (2011). Disjunctivism again. Philosophical Explorations, 14(1), 43-80.

Chemero, A. (2003). An outline of a theory of affordances. Ecological Psychology, 15, 181-95.

Chemero, A. (2009). Radical embodied cognitive science. Cambridge, MA: MIT Press.

Dreyfus, H. (2006). Overcoming the myth of the mental. Topoi, 25, 43-49. 
Duhem, P. (1991). The aim and structure of physical theory. New Jersey: Princeton University Press.

Evans, G. (1982). The varieties of reference. Oxford: Clarendon Press.

Favela, L. H., \& Chemero, A. (2016). An ecological account of visual “illusions”. Florida Philosophical Society, 16(1), 68-93.

Favela, L. H., Riley, M. A., Schokley, K., \& Chemero, A. (2018). Perceptually equivalent judgments made visually and via haptic sensory-substitution devices. Ecological Psychology 30(4), 326-345.

Gibson, J. J. (1967). New reasons for realism. Synthese, 17, 162-72.

Gibson, J. J. (1968). The senses considered as perceptual systems. London: George Allen \& Unwin.

Gibson, J. J. (1970). On the relation between hallucination and perception. Leonardo, 33, 425-427.

Gibson, J. J. (2015). The ecological approach to visual perception, classical edition. New York: Psychology Press.

Gibson, J. J., \& Gibson, E. (1955). Perceptual learning: Differentiation or enrichment? Psychological Review, 62, 32-41.

Haddock, A., \& Macpherson, F. (2008). Introduction: Varieties of disjunctivism. In A. Haddock \& F. Macpherson (Eds.), Disjunctivism: Perception, action, knowledge (pp. 1-24). Oxford: Oxford University Press.

Held, R., \& Hein, A. (1963). Movement-produced stimulation in the development of visually guided behavior. Journal of Comparative and Physiological Psychology, 56(5), 872-876.

Heras-Escribano, M., \& Pinedo, M. (2016). Are affordances normative? Phenomenology and the Cognitive Sciences, 15(4), 565-589.

Hinton, J. Visual experiences. Mind, LXXVI(302), 1967, 217-227.

Hutto, D. D., \& Myin, E. (2017). Evolving enactivism: Basic minds meet content. Cambridge, MA: MIT Press. 
Kuhn, T. (2012). The structure of scientific revolutions: 50th anniversary edition. Chicago: University of Chicago Press.

McClelland, T. (2015). Affording introspection: An alternative model of inner awareness. Philosophical Studies, 172, 2469-2492.

McDowell, J. (1982). Criteria, defeasibility, and knowledge. Proceedings of the British Academy, 68, 455-479.

McDowell, J. (1996). Mind and world. Cambridge: Harvard University Press.

McDowell, J. (2011). Perception as a capacity for knowledge. Milwaukee: Marquette University Press.

Michaels, C., \& Carello, C. (1981). Direct perception. New Jersey: Prentice-Hall.

Millar, A. (2007). What the disjunctivist is right about. Philosophy and Phenomenologial Research, LXXIV(1), 176-198.

Millar, A. (2009). What is it that cognitive abilities are abilities to do? Acta Analytica, 24(4), 223236.

Millar, A. (2016). Abilities, competences, and falibility. In M. Ángel \& F. Vargas (Eds.), Performance epistemology: Foundations and applications (pp. 62-82). Oxford: Oxford University Press.

Myin, E. (2016). Perception as something we do. Journal of Consciousness Studies, 23(5-6), 80104.

Noë, A. (2004). Action in perception. Cambridge, MA: MIT Press.

Noë, A. (2015). Concept pluralism, direct perception, and the fragility of presence. In T. Metzinger \& J. M. Windt (Eds.), Open MIND (pp. 1-15). Frankfurt am Main: MIND Group.

Pritchard, D. (2012). Epistemological disjunctivism. Oxford: Oxford University Press.

Pritchard, D. (2015). Epistemic angst. New Jersey: Princeton University Press. 
Rowlands, M. (2010). The new science of the mind: From extended mind to embodied phenomenology. Cambridge, MA: MIT Press.

Ryle, G. (1964). Dilemmas. London: Cambridge University Press.

Schellenberg, S. (2016). Perceptual particularity. Philosophy and Phenomenological Research, 93, 25-54.

Sellars, W. (1991). Science, perception and reality. Atascadero: Ridgeview;

Smith, A. D. (2000). Space and sight. Mind, 109, 481-518.

Snowdon, P. (2008). Hinton and the origins of disjunctivism. In A. Haddock \& F. Macpherson (Eds.), Disjunctivism: Perception, action, knowledge (pp. 35-56). Oxford: Oxford University Press.

Shaw, R., \& McIntyre, M. (1974). The role of symmetry in event perception. In R. MacLeod \& H. Pick (Eds.), Perception: Essays in honor of James J. Gibson (pp. 276-310). Ithaca: Cornell University Press.

Turvey, M. T. (1992). Affordances and prospective control: An outline of the ontology. Ecological Psychology, 4, 173-187.

Turvey, M. T., Shaw, R., Reed, E., \& Mace, W. M. (1981). Ecological laws of perceiving and acting: In reply to Fodor and Pylyshyn (1981)*. Cognition, 9(3), 237-304.

Warren, W. H. (1984). Perceiving affordances: Visual guidance of stair climbing. Journal of Experimental Psychology, Human Perception and Performance, 10, 683-703. 